\title{
International Journal of Advanced Biochemistry Research
}

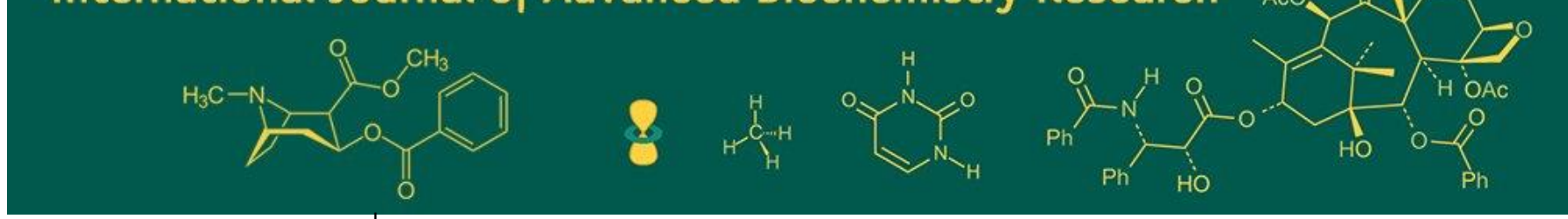

ISSN Print: $2617-4693$ ISSN Online: $2617-4707$ IJABR 2019; 3(2): 05-08 www.biochemjournal.com Received: 04-05-2019 Accepted: 08-06-2019

Dr. Manzura R Mulani IIMSR Medical College and Noor Hospital Campus, Aurangabad Jalna Highway, Warudi, Badnapur, Jalna, Maharashtra, India

Dr. Pandharinath S Gawali Professor, Medicine Department, IIMSR medical college and Noor hospital campus, Aurangabad Jalna highway, Warudi, Badnapur, Jalna, Maharashtra, India

Dr. Veena S. Hatolkar Professor, Biochemistry Department, IIMSR Medical College and Noor hospital Campus, Aurangabad Jalna Highway, Warudi, Badnapur, Jalna, Maharashtra, India
Corresponding Author: Dr. Manzura R Mulani IIMSR Medical College and Noor Hospital Campus, Aurangabad Jalna Highway, Warudi, Badnapur, Jalna, Maharashtra, India

\section{Study of biochemical markers in patients of ischemic heart disease and hypertension in IIMSR Hospital, Warudi}

\author{
Dr. Manzura R Mulani, Dr. Pandharinath S Gawali and Dr. Veena S \\ Hatolkar
}

\begin{abstract}
Introduction: Cardiovascular disease is the most frequent cause of adult death in industrial societies and is increasingly important in developing countries like India.

In the year 2000 the European Society of Cardiology and the American College of Cardiology Committee jointly redefined Myocardial Infarction (MI) by an elevation of cardiology troponin $\mathrm{T}$ (Trop $\mathrm{T}$ ) and cardiac troponin (cTn) is diagnostic and high sensitivity of cTn for detection of even small amounts of myocardial necrosis. Presently Troponin are the most prominent biomarker of cardiac injury is $1^{\text {st }}$ cardiac biomarker which a clinician can use at the bedside of the patient for risk stratification of cardiac injury and have relegated conventional biomarkers like CK-MB to $2^{\text {nd }}$ place. This is so because Cardiac Troponic are highly cardiac tissue specific unlike conventional biomarkers.

Materials and Methods: Diagnosed ischemic heart disease (IHD) and hypertensive (HTN) patients were taken as cases, out of these divided into two groups of patients. The first group $(n=50)$ were patients with IHD including MI. The second group $(n=50)$ were patients with HTN and third group $(\mathrm{n}=50)$ were normal healthy control. In all 3 groups cTnT \& CK-MB biochemical markers are evaluated.

Results: In all diseased two groups cTnT and CK-MB biochemical markers are elevated and in control healthy group CTnT \& CK-MB are normal.

Conclusion: Small elevations of trop T identify minimal cardiac necrosis and patients can benefit from early invasive therapy. Trop T and CK-MB had an independent effect on long term survival and the combination of both is associated with even worse survival.
\end{abstract}

Keywords: Ischemic heart disease, hypertension, Troponin T \& CK-MB

\section{Introduction}

Heart disease represents the major challenge that affects each of professionally and personally. Extensive research by man over several decades has concluded that cure of heart disease is very difficult, hence prevention of such ischemic events become mandatory.

Coronary heart disease also known as IHD is a group of disease that includes stable angina, unstable angina, MI \& sudden cardiac death ${ }^{[1]}$.

Cardiac troponin (C Tn) is highly sensitive and specific biochemical markers for myocardial necrosis and predicts increased risk of mortality and reinfarction in patients presenting with acute coronary syndrome (ACS) ${ }^{[2]}$.

Since 1994, a rapid and simple test has been available for the bedside determination of Trop $\mathrm{T}^{[3]}$.

In the last fifteen years use have gained confidence in their clinical utility as markers of myocardial necrosis which is generally due to ischemia. They are more sensitive and specific than conventional markers like CK-MB, lactate dehydrogenase (LDH) ${ }^{[4]}$.

According to World Health Organization (WHO) 1 billion people worldwide have HTN. Elevated trop $\mathrm{T}$ levels were also found to be a risk factor for the development of $\mathrm{LVH}$ in patients without baseline HTN \& clinical CVD ${ }^{[5]}$.

The biochemical markers should be measured during their "diagnostic window" and during this period trop T $100 \%$ sensitive ${ }^{[6]}$.

CK-MB typically is detected in the serum 4-6 hrs after the onset of ischemia. Ischemia may be caused by a mismatch between oxygen supply and demand at a cellular level ${ }^{[7]}$.

Frequency and severity of CVD, diagnosis must be rapid and accurate, diagnosis on an ECG 
is not absolute criterion, particularly in the case of small infarctions. Assays of plasma markers are thus of the importance to confirm the clinical hypothesis ${ }^{[8]}$.

ECG was one of the earliest diagnostic modalities introduced to evaluate ischemic injury to the heart. Dispite its usefulness, ECG continues to be non-sensitive in diagnosing ischemic cardiac events, as ECG finding are normal in many patients with ischemic injury.

The introduction of various markers of cardiac injury such as CK-MB, Troponins and myoglobin, has revolutionized the diagnosis of myocardial injury ${ }^{[9]}$.

HTN continuous to be potent and widespread risk factor for IHD. Among other risk factor, HTN plays an independent role in augmenting IHD risk, as well as a multiplicative role with respect to adverse outcomes when, HTN is present concurrently with the other major IHD risk factors ${ }^{[10]}$.

The present study aim to determine elevated Trop $\mathrm{T}$ have significantly prognostic implications and to the plasma concentrations of cTnT and CK-MB that correlate with myocardial ischemia.

The CK-MB isoenzyme levels predicted long term mortality independent of cTn. Troponin \& CK-MB had an independent effect on long term survival and the combination of both is associated with even worse survival [11].

On the other hand, the serum concentrations of cTnT, a marker of myocyte injury, can be used to risk stratifying patients presenting with chronic or acute heart failure.

Presently troponin, are the most prominent biomarkers of cardiac injury and have relegated conventional biomarkers like CK-MB \& LDH to $2^{\text {nd }}$ place. This is so because cardiac troponins are highly cardiac tissue specific unlike conventional biomarkers ${ }^{[4]}$.

This is $1^{\text {st }}$ cardiac biomarkers which a clinician can use at the bedside of the patient for risk stratification and diagnosis.CK-MB is an isoenzyme of $\mathrm{CK}$, which is relatively specific for myocardium. The decision to admit a patient with chest pain must ultimately be based on clinical factors including the evaluation of pain and the presence can however help identify such patients at high risk of infarction and death for whom earlier and more invasive investigation is indicated ${ }^{[6]}$.

There are several potential advantages of bedside measurement of biochemical markers with the new assay and reader device with chest pain. A biochemical tests immediately available for physicians and may thus aid in the triage of chest pain.

Both clinical chemistry laboratory assays and bedside assays are now available to measure several biochemical cardiac markers notable the CK-MB \& $\mathrm{cTnT}^{[12]}$.

\section{Materials \& Methods}

The present study was conducted in JIIU'S IIMSR medical college and hospital, Warudi, dist-Jalna, Maharashtra, India. During the period from March 2017 to February 2018. The patients were taken from the department of medicine, 33 (24 males \& 9 females) diagnosed IHD patients were taken from the department of medicine, $n=50$ diagnosed IHD patients of both sex with age range of 39-65 years were taken as group I, n=50 were taken as only HTN as group II and $n=50$ is healthy control taken as group III. Cases were the diagnosed positive ECG finding IHD patients of both sex admitted in the hospital during the study period.

We prespecified comparisons of studies enrolling patients with suspected ischemia versus studies enrolling patients in whom HTN has diagnosed.

We investigate biochemical markers such as Trop $\mathrm{T}$ and CK-MB in all the above three groups.

Overall patients with a positive trop $\mathrm{T}$ had significantly worse outcomes than patients with a negative trop $\mathrm{T}$. The determination with trop $\mathrm{T}$ sensitive (Roche Diagnostics GMbH, Mannhein Gemany) were carried with EDTA blood using 2 test lots. The test was performed as specified. CKMB was determined by immunoinhibition method.

\section{Results}

Consecutive patients admitted in the $24 \mathrm{hrs}$ after the onset of chest pain were enrolled in the study. Tests of bedside cTnT qualitative, CK-MB \& ECG were performed on these patients.

CK-MB levels were found to be elevated in samples in $60 \%$ of cases after the onset of chest pain.

The association of cTnT positive with significantly higher CK-MB as compared to negative cTnT predisposes the patient to greater risk of serious outcome.

Our study reveals only $54 \%$ cTnT positive in IHD subjects and $24 \%$ in HTN subjects. While have an episode of microinfarction where cTnT measurements and yet CK-MB even by mass assay remains in the normal range.

Bonferroni test was applied for multiple comparisons between CK-MB IHD, CK-MB HTN \& CK-MB control.

Mean of SD CK-MB (23.57 \pm 6.30$)$ in IHD patients, mean SD of CK-MB $(22.16 \pm 6.23)$ in HTN patients and mean SD of CK-MB (14.56 \pm 4.90$)$ in control group.

In group I, patients with IHD significantly higher mean CKMB levels when compared to healthy controls and highly significant $(\mathrm{P}=0.000)$ in Table no. 1 .

In group II, patients with HTN significantly higher mean CK-MB levels when compared to healthy control and are highly significant. $(\mathrm{P}=0.000)$ in Table no. 1 .

Table 1: Mean SD CK-MB values in IHD, HTN and healthy control group

\begin{tabular}{|c|c|c|c|c|}
\hline Parameter $(\mathbf{n g} / \mathbf{m l})$ & Control $(\mathbf{n}=\mathbf{5 0})$ & IHD patients $(\mathbf{n = 5 0})$ & HTN patients $(\mathbf{n = 5 0})$ & significance \\
\hline CK-MB & $14.56 \pm 4.9$ & $23.57 \pm 6.30$ & $22.16 \pm 6.2$ & 0.000 \\
\hline
\end{tabular}

\section{Discussion}

CTnT are located on myofibrillar thin (actin) filament of striated (skeletal and cardiac) muscle. Hence CTn is contractile protein more specific than $\mathrm{CK}$ values for myocardial injury and because of their high sensitivity ${ }^{[13]}$.

It is clinically important to be able to diagnose episodes of microinfarction that are below the "radar screen" provided by CK-MB? Let us $1^{\text {st }}$ look at the answer to this question as on might view a black and white photograph that is a dichotomous analysis of trop $\mathrm{T}$ tests the results declared as positive (above the decision limit) or negative (below the decision limit) ${ }^{[14]}$.

Small rise in troponin levels may have as markers for subclinical high blood pressure and heart damage, more sensitive version of a damage from heart attacks could also identify peoples on their way to developing HTN well before the so called 'silent killer' shows up on a B.P. machine ${ }^{[15]}$. 
Patients with a normal CK-MB level but elevated troponin levels are considered to have sustained minor myocardial damage or microinfarction, where as patients with elevations of both CK-MB \& troponin are considered to have had AMI. The cardiac troponin may remain elevated up to 2 weeks after symptoms onsets, which makes the useful as late markers of recent AMI ${ }^{[16]}$.

However in the majority of patients presenting with EHT and free from HF, the TnT concentrations are $<0.01 \mathrm{ng} / \mathrm{ml}$, thus are undetectable by conventional TnT assay. A high sensitive qualitative assay recently available and detect very small amount of TnT released into the circulation of HTN patients in the absence of $\mathrm{HF}^{[17]}$.

Everett et al. reported that an abnormal of cTnT was an independent predictor of $\mathrm{CV}$ events in elderly patients with stable IHD ${ }^{[18]}$.

The mortality in patients with a positive troponin was $5.2 \%$ compared with the mortality in patients with a negative troponin of $1.6 \%$

The overview found that measurement of serum troponin provides significant information about the short term risk of death for patients with non-ST segment elevation acute coronary syndrome and positive troponin have short term risk of death 3 to 8 fold higher than patients with a negative test ${ }^{[19]}$.

Cardiac troponins have been in use for evaluation of patients with chest pain and ACS for the last 15 years. They have rapidly attained central role in diagnosis, prognostication and planning of therapeutic strategies in these patients ${ }^{[4]}$.

Positive troponins is indicative of myocardial necrosis, because it is well established that, LV function is a pivotal determinant of prognosis, it is possible that loss of functioning myocardium is associated with a worse outcome [14].

cTn interact with tropomycin to form the troponintropomycin complex. This complex forms the skeleton of the striated muscle and has a regulator function in the excitation contraction coupling of the heart. If heart muscle cells are damaged by acute ischemia or any other mechanism these proteins are released into the blood stream. [20].

Researcher said the standard trop $\mathrm{T}$ already the gold standard screen for cardiac muscle damage from ongoing or recent heart attacks work great for figuring out whether someone with chest pain or other cardiac symptoms is having a heart attack, but results often come back "normal" for many with other forms of cardiac damage untreated to heart attacks.

The high sensitivity test used in the study can identify these people, the researcher add because it detects even trace amounts of troponin released by heart cells injured by spiked in B.P. that come and go un-noticed.

HBP's stealthy onset, it variability over time and the need to recheck its over multiple visits before making a definitive diagnosis have hampered efforts to treat a condition that is a leading cause of heart attacks, strokes and claims greater than 9 million lives worldwide each year's according to $\mathrm{WHO}^{[5]}$.

Keffer has proposed a practice guideline for acute IHD \& the National Academy of clinical Biochemistry has also published recommendations for the use of cardiac markers. [21].

CK-MB has reported as a specific and sensitive marker of both clinical and subclinical myocardial injury, the CK-MB extent of the elevation in serum in serum depends on the severity of the myocardial injury ${ }^{[22]}$.

Patients with elevated troponin level but negative CK-MB values who were formerly diagnosed with unstable angina or minor myocardial injury are now reclassified as non-ST segment elevation MI even in the absence of diagnostic ECG changes ${ }^{[23]}$.

\section{Conclusion}

Elevated cTn values provide prognostic information and seens to the severity of the disease process. Trop $\mathrm{T}$ useful in the emergency department who could be safety discharged home.

cTnT may be more sensitive to detect minor infarction which could not be detected by CK-MB.

Troponin measurement was "sold" to the cardiology and emergency medicine physicians as the "golden tricket."

TropT and CK-MB considered as the "gold standard" for MI diagnosis, these patients should go for at least for 1 year with these novel markers.

Hs-cTnT level as an indicator of subclinical ongoing myocyte damage could be a good novel marker to predict and /or indicate deteriorating cardiac remodeling and LV diagnostic dysfunction in HTN.

\section{References}

1. Ganesh Ghuge D, Rahul Zine. Mukund Mogrekar Lipid profile of patients with ischaemic heart disease from rural area of marthawada region, Int $\mathrm{J}$ of Biomedical and Advance Research. 2012; 03(10):767-69.

2. Giora Landesberg, Vadim Shatz, Inna Akopnik et al. Association of cardiac troponin, CK-MB and postoperative myocardial ischemia with long-term survival after major vascular surgery. Journal of the American College of Cardiology. 2003; 42(9):1547-54.

3. Harald Herkner, Paul Collinson O et al. Analytical and Clinical Performance of an Improved Qualitative Troponin T Rapid Test in Laboratories and Critical Care Units, Archives of pathology \& laboratory medicine. 2000; 124:283-87.

4. Takeda S, Yamashita A, Maeda K et al. Structure of the core domain of human cardiac troponin in the $\mathrm{Ca}(2+)$ saturated form. Nature, 2003; 424:35-41.

5. McEvoy J, Chen Y, Nambi V, Ballantyne CM et al. High-Sensitivity Cardiac Troponin $\mathrm{T}$ and Risk of Hypertension. Circulation, 2015.

6. Huggon AM, Chambers $\mathrm{J}$ et al. Biochemical markers in the management of suspected acute myocardial infarction in the emergency department; Emerg Med J. 2001; 18:15-19.

7. Kristopher Lyons S, Gareth McKeeman et al. Highsensitivity troponin $\mathrm{T}$ is detectable in most patients with clinically stable heart failure. Br J Cardiol. 2014; 21:33-6.

8. Javad Mohiti, Mostafa Behjati, Mohammad Soltani H, Ali Babaei. The significance of troponin T and CK-MB release in coronary artery bypass surgery, Indian Journal of Clinical Biochemistry. 2004; 19(1):113-117.

9. Ashvarya Mangla, Eric Staros B. Troponins; Medscape, news \& perspective drugs \& diseases CME \& education academy; Update, 2015, 14.

10. Agbor-Etang BB, Setaro JF. Management of Hypertension in Patients with Ischemic Heart Disease. Curr Cardiol Rep. 2015; 17(12):119. 
11. Giora Landesberg, Vadim Shatz et al. Association of Cardiac Troponin, CK-MB, and Postoperative Myocardial Ischemia With Long-Term Survival After Major Vascular Surgery, Journal of the American College of Cardiology. 2003; 42(9):1547-54.

12. Quantitative Bedside Assay for Cardiac Troponin T: A Complementary Method to Centralized Laboratory Testing; Clinical Chemistry. 1999; 45(7):1002-1008.

13. Susanne Korff, Hugo Katus A, Evangelos Giannitsis. Differential Diagnosis of Elevated Troponins, Heart. 2006; 92:987-993.

14. Elliott Antman M, Boston Massachusetts. Troponin Measurements in Ischemic Heart Disease: More Than Just a Black and White Picture Journal of the American College of Cardiology. 2001; 38(4):987-90.

15. John McEvoy W, Yuan Chen, Vijay Nambi, Christie Ballantyne M et al. High-Sensitivity Cardiac Troponin $\mathrm{T}$ and Risk of Hypertension. Circulation, 2015.

16. Suraj Achar A, Suriti Kundu et al. diagnosis of acute coronary syndrome, American family physician. 2005; 72(1):119-126.

17. Yukihito Sato, Erika Yamamoto et al. High-sensitivity cardiac troponin $\mathrm{T}$ in Essential Hypertension, Journal of Cardiology. 2011; 58:226-231.

18. Everett BM, Brooks MM, Vlachos HEA, Chaitman BR, Frye RL, Bhatt DL. Troponin and cardiac events in stable ischemic heart disease and diabetes. N Engl J Med. 2015; 373:610-20.

19. Paul Heidenreich A, Thomas Alloggiamento et al. The Prognostic Value of Troponin in Patients with Non-ST Elevation Acute Coronary Syndromes: A MetaAnalysis, Journal of the American College of Cardiology. 2001; 38(2):478-85.

20. Asli Tanindi, Mustafa Cemri. Troponin elevation in conditions other than acute coronary syndromes, Vascular Health and Risk Management. 2011; 7:597603.

21. Ana Bennett E, Roger Bertholf L. Discordant Results of CK-MB and Troponin I Measurements: A Review of 14 Cases, nnals of Clinical \& Laboratory Science. 2000; 30(2):167-73.

22. Inaam Amin A. The Effect of Atenolol on CK-MB Levels in Hypertensive Patients, Iraqi J Pharm. 2009; 18(1):1-4.

23. Donald Schreiber, Cardiac Markers, The heart Medscape, Updated, 2018. 